\section{The canonical Kravchuk basis for discrete quantum mechanics}

To cite this article: Tugrul Hakioglu and Kurt Bernardo Wolf 2000 J. Phys. A: Math. Gen. 333313

View the article online for updates and enhancements.

\section{Related content}

- The action-angle Wigner function
T Hakioglu and E Tepedelenlioglu
- Finite two-dimensional oscillator: I. The
Cartesianmodel
Natig M Atakishiyev, George S Pogosyan,
Luis Edgar Vicent et al.
- Linear canonical transformations and
quantum phase:a unified canonical and
algebraic approach

\section{Recent citations}

- Apostolos Vourdas

A symmetric generalization of

Sturm-Liouville problems in discrete spaces

M. Masjed-Jamei and I. Area

On limit relations between some families of bivariate hypergeometric orthogonal polynomials

I Area and E Godoy 


\title{
The canonical Kravchuk basis for discrete quantum mechanics
}

\author{
Tuğrul Hakioğlu† and Kurt Bernardo Wolf \\ Centro Internacional de Ciencias, Avenida Universidad 1001, 62210 Cuernavaca, Mexico
}

Received 7 December 1999

\begin{abstract}
The well known Kravchuk formalism of the harmonic oscillator obtained from the direct discretization method is shown to be a new way of formulating discrete quantum phase space. It is shown that the Kravchuk oscillator Hamiltonian has a well defined unitary canonical partner which we identify with the quantum phase of the Kravchuk oscillator. The generalized discrete Wigner function formalism based on the action and angle variables is applied to the Kravchuk oscillator and its continuous limit is examined.
\end{abstract}

\section{Introduction}

A satisfactory model of discrete and finite quantum mechanics can be based on correspondingly discrete and finite phase space. The formulation of quantum mechanics on a finite-dimensional phase space has commonly used the bases natural to the Fourier transform: circular functions and Kronecker $\delta$. This approach, for which some very early references exist [1], implies that phase space is a toroidal lattice; the dynamical operators are elements of the discrete unitary Weyl-Heisenberg group [2,3]. In this paper, we use the Kravchuk functions [4], the discrete and finite quantum harmonic oscillator functions studied by Atakishiyev and Suslov [5], and recently used for optical models [6], to provide a pair of canonically conjugate Weyl-Heisenberg operators. The advantage afforded by the Kravchuk basis over the Fourier basis is that the Weyl-Heisenberg dynamical operators are those of the harmonic oscillator, rather than those of the free particle.

In section 2 we follow the discretization of the harmonic oscillator Hamiltonian in the way of Atakishiyev and Suslov [5]. The solutions of the discrete Schrödinger equation are special orthogonal polynomials, with orthogonality and completeness relations on a discrete set of points [4]. Particularly for the discrete and finite harmonic oscillator, the solutions are Kravchuk functions [6]: the ground state is the square root of the binomial distribution (which is in the class of 'discrete Gaussians'), and the Hamiltonian has an equidistant quantized energy spectrum, as the standard harmonic oscillator-but finite. These functions have recently been used to describe an $S U$ (2) group of optical Fourier-like transformations and, in particular, the fractional Fourier-Kravchuk transformation [6]. This basis, its Fourier-Kravchuk, and its standard Fourier transform are described in section 3.

Section 4 reviews the construction of the discrete, unitary Weyl-Heisenberg-Schwinger representation of the canonical phase space operators [2,3], which is now possible in the Kravchuk basis, for a discrete subgroup of the Fourier-Kravchuk transform. The discrete

$\dagger$ Corresponding author. Present address: Physics Department, Bilkent University, 06533 Ankara, Turkey.

$\ddagger$ Permanent address: Centro de Ciencias Físicas, Universidad Nacional Autónoma de México Cuernavaca, 62251 Cuernavaca, Mexico. 
Wigner function of the action-angle variables is computed for the Kravchuk basis in section 5 . It is shown to fulfill expectations for the action and angle intepretations of its marginal distributions, including the case of split ('Schrödinger cat') states. In section 6 we build the quantum phase operator for the Kravchuk oscillator and examine its time evolution. The time evolution of the action-angle Wigner function is also examined. The continuum limit is addressed in section 7 for the action-angle variables in the discrete Wigner function, for which it is a proper limit. We summarize some conclusions in section 8 .

\section{The Kravchuk oscillator}

In this section we will recapitulate the discretization of the quantum harmonic oscillator, as formulated by Atakishiyev and Suslov in [5], and introduce the Kravchuk basis [4].

The stardard integrable solutions of the harmonic oscillator Schrödinger equation in the $x$-representation (in the range $-\infty<x<\infty$ ),

$$
-\mathrm{i} \frac{\partial}{\partial t} \Psi(x ; t)=\frac{1}{2}\left(-\frac{\partial^{2}}{\partial x^{2}}+x^{2}\right) \Psi(x ; t)=\mu_{n} \Psi(x ; t)
$$

are found as $\Psi_{n}(x ; t)=\mathrm{e}^{\mathrm{i} \mu_{n} t} \Psi_{n}(x)$ in terms of the well known Hermite functions and eigenvalues

$$
\Psi_{n}(x)=\frac{1}{\sqrt{\sqrt{\pi} 2^{n} n !}} \mathrm{e}^{-x^{2} / 2} H_{n}(x) \quad \mu_{n}=n+\frac{1}{2}
$$

where $\mu_{n}$ are the energy eigenvalues in units of $\hbar \omega$.

To formulate the direct discretization of (1), we recall that the classical Hermite orthogonal polynomials are solutions of the differential equation

$$
\frac{\mathrm{d}^{2} y_{n}(x)}{\mathrm{d} x^{2}}-2 x \frac{\mathrm{d} y_{n}(x)}{\mathrm{d} x}+\lambda_{n} y_{n}(x)=0 \quad \lambda_{n}=2 n
$$

with $n=0,1,2, \ldots$ Now consider a discrete subset of points $x$ in steps of $h$, and write $x \mapsto x_{k}=h k+x_{o}$, with $k$ integer and $x_{o}$ arbitrary, but conveniently put to zero. Correspondingly in (3), we write

$$
\begin{aligned}
& \frac{\mathrm{d} f(x)}{\mathrm{d} x} \mapsto \Delta f\left(x_{k}\right)=\frac{1}{h}\left(f\left(x_{k+1}\right)-f\left(x_{k}\right)\right) \\
& \frac{\mathrm{d}^{2} f(x)}{\mathrm{d} x^{2}} \mapsto \Delta \nabla f\left(x_{k}\right) \quad \nabla f\left(x_{k}\right)=\Delta f\left(x_{k-1}\right) .
\end{aligned}
$$

After substitution and for $h=1$, (3) gives rise to a second-order difference equation which relates the values of the solution at three equidistant points.

From the Hermite differential equation one obtains the Kravchuk difference equation whose solutions are the Kravchuk polynomials

$$
k_{n}\left(x_{k}, D\right)=\frac{(-1)^{n}}{2^{n}}\left(\begin{array}{l}
D \\
n
\end{array}\right){ }_{2} F_{1}(-n,-k ;-D ; 2)
$$

for $D=0,1,2, \ldots$ These are continuously defined for complex $x$, as all polynomials are, but they satisfy the discrete orthogonality and completeness relations

$$
\begin{aligned}
& \sum_{k=0}^{D-1} \rho\left(x_{k}\right) k_{n}\left(x_{k}, D\right) k_{m}\left(x_{k}, D\right)=d_{n}^{2} \delta_{n, m} \\
& \sum_{n=0}^{D-1} \frac{1}{d_{n}^{2}} k_{n}\left(x_{k}, D\right) k_{n}\left(x_{\ell}, D\right)=\rho\left(x_{k}\right) \delta_{k, \ell}
\end{aligned}
$$


where the discrete measure and the normalization constant are binomial distributions,

$$
\rho\left(x_{k}\right)=\frac{(D-1) !}{2^{(D-1)} x_{k} !\left(D-1-x_{k}\right) !} \quad d_{n}^{2}=\frac{(D-1) !}{4^{n} n !(D-1-n) !} .
$$

The orthogonality relation is proven in a manner similar to the partial integration argument used for the classical orthogonal polynomials. Note that the measure is nonzero for integer $0 \leqslant x_{k}=k \leqslant D-1$, and the normalization is nonzero for $0 \leqslant n \leqslant D-1$.

The Kravchuk functions $K_{n}\left(x_{k}, D\right)$ are then built [6] to be orthonormal and complete with respect to a unit measure. Thus,

$$
K_{n}\left(x_{k}, D\right)=\frac{1}{d_{n}} \sqrt{\rho\left(x_{k}\right)} k_{n}\left(x_{k}, D\right)
$$

are a basis for the $D$-dimensional space of vectors. They are analytic on the slightly larger interval $(-1, D)$ (with branch-point zeros at the ends), and satisfy orthogonality relations on the integers contained therein,

$$
\sum_{k=0}^{D-1} K_{n}\left(x_{k}, D\right) K_{m}\left(x_{k}, D\right)=\delta_{n, m}=\sum_{k=0}^{D-1} K_{k}\left(x_{n}, D\right) K_{k}\left(x_{m}, D\right) .
$$

Their time-dependent discrete Schrödinger equation [5] can be found using (5) and multiplying (3) by $\rho\left(x_{k}\right)$. One obtains

$$
-\mathrm{i} \frac{\partial}{\partial t} K_{n}\left(x_{k}, D ; t\right)=\hat{\mathcal{H}}_{D} K_{n}\left(x_{k}, D ; t\right)=\mu_{n} K_{n}\left(x_{k}, D ; t\right)
$$

where the discrete harmonic oscillator Hamiltonian is the difference operator with an equally spaced spectrum corresponding to unit frequency,

$\sqrt{\rho\left(x_{k}\right)} \hat{\mathcal{H}}_{D} \frac{1}{\sqrt{\rho\left(x_{k}\right)}}=-\Delta\left(\sigma\left(x_{k}\right) \rho\left(x_{k}\right) \nabla \frac{1}{\rho\left(x_{k}\right)}\right)+\frac{1}{2} \quad \mu_{n}=n+\frac{1}{2}$

where $\sigma\left(x_{k}\right)=x_{k}$ here (we retained the standard general notation adopted in [4]). The time dependence of the Kravchuk solutions of the discrete oscillator is $K_{n}\left(x_{k}, D ; t\right)=$ $\mathrm{e}^{\mathrm{i} \mu_{n} t} K_{n}\left(x_{k}, D\right)$, as was the case with the Hermite functions. The centre of the oscillator is at the midpoint between 0 and $D$, namely at $x=\frac{1}{2} D$; when $D$ is even, this coincides with a point in the set $x_{k}$.

In figure 1 we plot a few of Kravchuk functions $K_{n}\left(x_{k}, D\right)$ for $D=61$, joining the values at the integers by straight lines. The lowest- $n$ functions visibly resemble the harmonic oscillator wavefunctions; the highest- $n$ plots relate to the lowest ones through the symmetry relation $K_{D-1-n}\left(x_{k}, D\right)=(-1)^{k} K_{n}\left(x_{k}, D\right)$. The Kravchuk functions also have definite parity: $K_{n}\left(D-1-x_{k}, D\right)=(-1)^{n} K_{n}\left(x_{k}, D\right)$. Finally, in the limit $D \rightarrow \infty$, the Kravchuk functions (10) approach the continuous Hermite-Gaussians (2),

$$
\lim _{D \rightarrow \infty}\left(\frac{1}{2} D\right)^{1 / 4} K_{n}\left(\frac{1}{2} D+\sqrt{\frac{1}{2} D} x, D\right)=\Psi_{n}(x) .
$$

The Kravchuk functions for fixed $D$ are also the unitary irreducible representation matrix elements of the angular momentum group $S U(2)$ for spin $\ell=\frac{1}{2}(D-1)[4,5,7]$. The $D \rightarrow \infty$ limit is then described by an Inönü-Wigner contraction of the $S U(2)$ generators to those of the Heisenberg algebra.

\section{The Fourier-Kravchuk transform}

The equidistance of the spectrum of the discrete Hamiltonian $\hat{\mathcal{H}}_{D}$ in the Kravchuk basis, (13), permits its use to define a fractional Fourier-like operator $\hat{\mathcal{F}}_{K}$, with all the desirable properties 
of (but distinct from) the standard Fourier transform $\hat{\mathcal{F}}$. This is the fractional Fourier-Kravchuk operator [6],

$$
\hat{\mathcal{F}}_{K}^{\alpha}=\exp \left(\frac{\pi}{2} \mathrm{i} \alpha\left(\hat{\mathcal{H}}_{D}-\frac{1}{2}\right)\right) .
$$

The Fourier-Kravchuk operators are unitary, and elements of a one-parameter cyclic group parametrized by real $\alpha$ modulo 4,

$$
\begin{array}{ll}
\hat{\mathcal{F}}_{K}^{\alpha_{1}} \hat{\mathcal{F}}_{K}^{\alpha_{2}}=\hat{\mathcal{F}}_{K}^{\alpha_{1}+\alpha_{2}} & \text { (composition) } \\
\left(\hat{\mathcal{F}}_{K}^{\alpha}\right)^{\dagger}=\hat{\mathcal{F}}_{K}^{-\alpha}=\left(\hat{\mathcal{F}}_{K}^{\alpha}\right)^{-1} & \text { (unitarity and inverse) }
\end{array}
$$

The desirable Fourier-like properties occur for $\alpha \in\{0,1,2,3\}=\mathbb{Z}_{4}$, and are

$$
\begin{aligned}
& \hat{\mathcal{F}}_{K}^{4}=\hat{\mathcal{F}}^{0}=\boldsymbol{I} \quad \text { (identity) } \\
& \hat{\mathcal{F}}_{K}^{2}=\hat{\mathcal{P}} \quad \text { (parity). }
\end{aligned}
$$

The parity operator $\mathcal{P}$ in (17) multiplies $K_{n}$ by $(-1)^{n}$, and thus has the same effect as the inversion of the function in $x_{k}$ through $\frac{1}{2}(D-1)$. The eigenvalues of the Fourier-Kravchuk operator $(15)$ are $\mathrm{e}^{\frac{\pi}{2} \mathrm{i} n} \in\{1, \mathrm{i},-1,-\mathrm{i}\}$ for $0 \leqslant n \leqslant D-1$, so their multiplicities can be put in terms of $N=0,1,2, \ldots$, distinguishing the cases by $D$ modulo 4 , as follows:

\begin{tabular}{l|llll}
$D$ & 1 & $\mathrm{i}$ & -1 & $-\mathrm{i}$ \\
\hline $4 N$ & $N$ & $N$ & $N$ & $N$ \\
$4 N+1$ & $N+1$ & $N$ & $N$ & $N$ \\
$4 N+2$ & $N+1$ & $N+1$ & $N$ & $N$ \\
$4 N+3$ & $N+1$ & $N+1$ & $N+1$ & $N$
\end{tabular}

(Fourier-Kravchuk).

The Fourier-Kravchuk transform should be compared with the Fourier transform operator $\hat{\mathcal{F}}$ in the standard $D \times D$ matrix representation, whose elements are powers of the $D$ th root of unity $\omega=\mathrm{e}^{-2 \pi \mathrm{i} / D}, \omega^{D}=1$,

$$
\hat{\mathcal{F}} \mapsto \frac{1}{\sqrt{D}}\left(\begin{array}{ccccc}
1 & 1 & 1 & \cdots & 1 \\
1 & \omega & \omega^{2} & \cdots & \omega^{D-1} \\
1 & \omega^{2} & \omega^{4} & \cdots & \omega^{2(D-1)} \\
\vdots & \vdots & \vdots & \ddots & \vdots \\
1 & \omega^{D-1} & \omega^{2(D-1)} & \cdots & \omega^{(D-1)^{2}}
\end{array}\right)=\frac{\left\|\omega^{k l}\right\|}{\sqrt{D}} .
$$

This matrix is unitary, symmetric and satisfies the properties (17). Its eigenvalues are also in the set $\{1, i,-1,-i\}$, and with multiplicities which also grow in a modulo-4 fashion, but of the form

\begin{tabular}{l|llll}
$D$ & 1 & $\mathrm{i}$ & -1 & $-\mathrm{i}$ \\
\hline $4 N$ & $N+1$ & $N$ & $N$ & $N-1$ \\
$4 N+1$ & $N+1$ & $N$ & $N$ & $N$ \\
$4 N+2$ & $N+1$ & $N$ & $N+1$ & $N$ \\
$4 N+3$ & $N+1$ & $N+1$ & $N+1$ & $N$
\end{tabular}

(Fourier).

For even $D$, the multiplicities of the Fourier and the Fourier-Kravchuk matrices are different. This is a strong selection rule against the existence of a unitary transformation between the $\left(\hat{\mathcal{F}}_{K}\right)_{D}$ and $(\hat{\mathcal{F}})_{D}$ for even dimensions.

The discrete Fourier transforms of the Kravchuk functions with respect to the energy index are given by

$$
H_{\ell}\left(x_{k}, D\right)=\left(\hat{\mathcal{F}} K_{\ell}\right)\left(x_{k}, D\right)=\frac{1}{\sqrt{D}} \sum_{n=0}^{D-1} \omega^{-n \ell} K_{n}\left(x_{k}, D\right)
$$



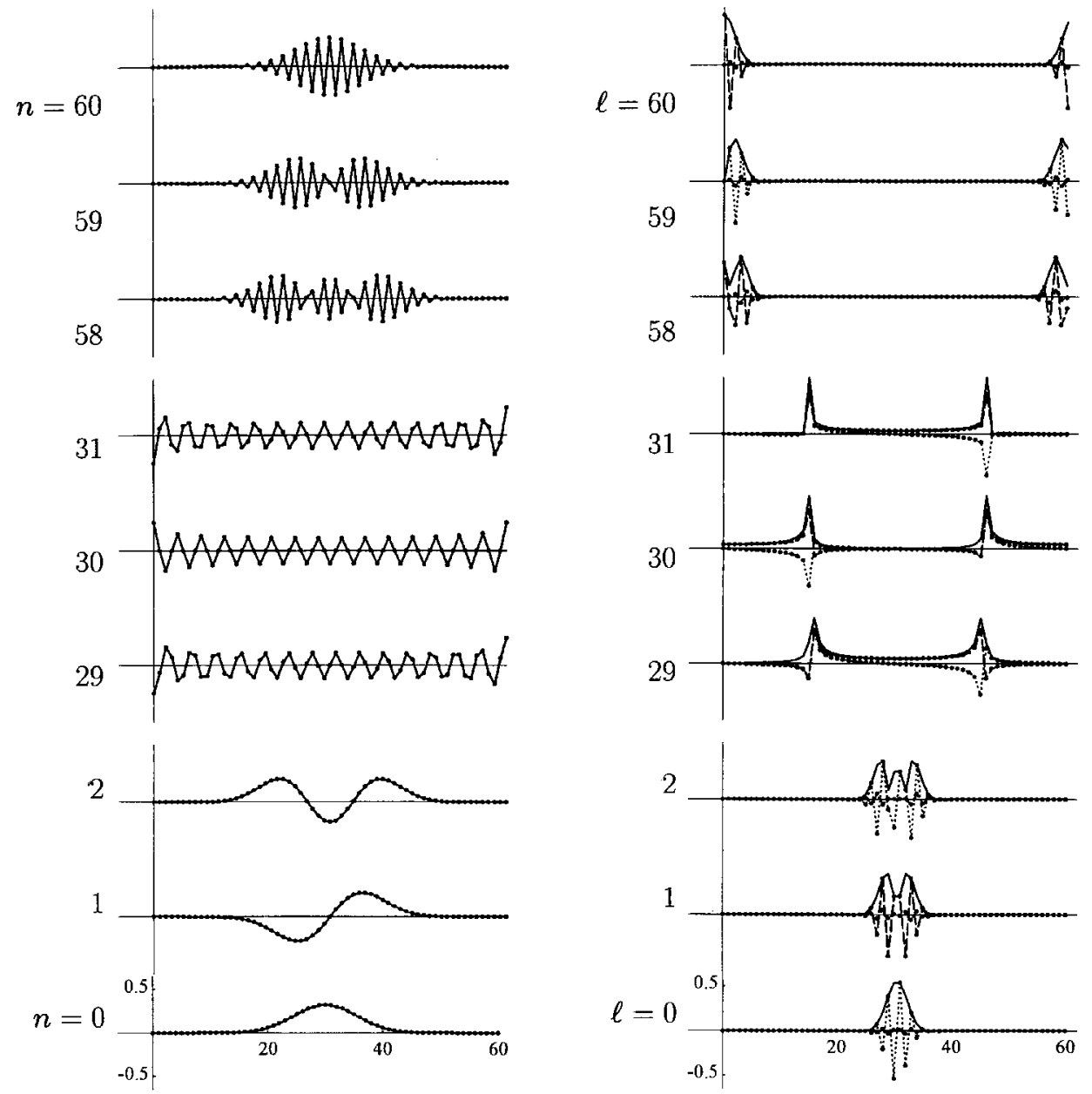

Figure 1. Kravchuk functions $K_{n}\left(x_{k}, D\right)$ given by (10) and (6) for $D=61$. From bottom to top, energy index $n=0,1,2, \ldots, 29,30,31, \ldots, 58,59,60$. The Kravchuk functions are plotted for the integer values of $x_{k}, k=0,1,2, \ldots, 60$.

Figure 2. Fourier transforms of the Kravchuk functions with respect to the energy index, $H_{\ell}\left(x_{k}, D\right)$ given by (19) for $D=61$. From bottom to top, $\ell=$ $0,1,2, \ldots, 29,30,31, \ldots, 58,59,60$; the functions are plotted for integer values of $x_{k}, k=0,1,2, \ldots, 60$.

These are plotted in figure 2 in the order corresponding to figure 1 . We want to emphasize that we could not find a 'closed' expression for these functions (19). Indeed, several linear and bilinear generating functions for Kravchuk polynomials can be found in the literature [4, 8], but not the one needed for (19). This equation therefore defines what are probably new special functions, which indeed are analytic on the interval $(-1, D)$ and are complete and orthonormal on the contained integers, namely

$$
\sum_{k=0}^{D-1} H_{n}\left(x_{k}, D\right) H_{m}\left(x_{k}, D\right)=\delta_{n, m}=\sum_{k=0}^{D-1} H_{k}\left(x_{n}, D\right) H_{k}\left(x_{m}, D\right)
$$




\section{The finite Kravchuk canonical pairs of operators}

In this section we summarize the construction of the analogue of the discrete unitary WeylHeisenberg-Schwinger representations of the canonical phase space [2,3], but in the discrete Kravchuk basis.

We begin by noting that the set of fractional Fourier-Kravchuk operators $\hat{\mathcal{F}}_{K}^{\alpha}, 0 \leqslant \alpha<4$ given in (15), have a finite subgroup, $C_{D}$ of order $D$, for discrete $\alpha=\alpha_{r}=4 r / D$ with $0 \leqslant r \leqslant(D-1)$. In the Kravchuk basis, the matrix representing $\hat{\mathcal{F}}_{K}^{\alpha_{r}}$ is diagonal,

$$
\hat{\mathcal{F}}_{K}^{\alpha_{r}} \mapsto \operatorname{diag}\left(1, \omega^{r}, \omega^{2 r}, \ldots, \omega^{(D-1) r}\right) \quad \omega=\exp (-2 \pi \mathrm{i} / D) .
$$

We define the unitary canonical partner $\hat{\mathcal{O}}^{r}$ of $\hat{\mathcal{F}}_{K}^{\alpha_{r}}$ through the standard Fourier transformation $\hat{\mathcal{F}}$ in (18), as

$$
\hat{\mathcal{O}}^{r}=\hat{\mathcal{F}} \hat{\mathcal{F}}_{K}^{\alpha_{r}} \hat{\mathcal{F}}^{-1} .
$$

It is represented by the matrix of elements

$$
\left(\hat{\mathcal{O}}^{r}\right)_{k, \ell}=\frac{1}{D} \sum_{m=0}^{D-1} \omega^{m(k-\ell+r)}=\delta_{k+r, \ell}
$$

where we consider the indices counted modulo $D$, i.e. $k, r, \ell \in \mathbb{Z}_{D}$. We note that $\omega^{D}=\hat{\mathcal{F}}_{K}^{\alpha_{D}}=$ $\hat{\mathcal{O}}^{D}=1$.

Using (21) and (23) one can prove that

$$
\hat{\mathcal{F}}_{K}^{\alpha_{k_{1}}} \hat{\mathcal{O}}^{k_{2}}=\omega^{k_{1} k_{2}} \hat{\mathcal{O}}^{k_{2}} \hat{\mathcal{F}}_{K}^{\alpha_{k_{1}}} \quad k_{1}, k_{2} \in \mathbb{Z}_{D}
$$

namely that $\hat{\mathcal{F}}_{K}^{\alpha_{k_{1}}}$ and $\hat{\mathcal{O}}^{k_{2}}$ are elements of the $D$-dimensional Weyl-Heisenberg group. As a result of the Fourier transform in (22), the eigenfunctions of $\hat{\mathcal{O}}$ are spanned by the set of functions $H_{\ell}\left(x_{k}, D\right)$ in (19).

The two orthonormal sets of functions $K_{n}\left(x_{k}, D\right)$ and $H_{\ell}\left(x_{k}, D\right)$ are discrete functional representations of the $D$-dimensional vector bases $\left\{\left|K_{n}\right\rangle\right\}_{0 \leqslant n \leqslant(D-1)}$ and $\left\{\left|H_{\ell}\right\rangle\right\}_{0 \leqslant \ell \leqslant(D-1)}$, where we find it advantageous to use the finite-dimensional Dirac ket notation. We thus have

$$
\begin{array}{ll}
\hat{\mathcal{F}}_{K}^{\alpha_{r}}\left|K_{n}\right\rangle=\omega^{n r}\left|K_{n}\right\rangle & \hat{\mathcal{F}}_{K}^{\alpha_{r}}\left|H_{\ell}\right\rangle=\left|H_{\ell-r}\right\rangle \\
\hat{\mathcal{O}}^{r}\left|K_{n}\right\rangle=\left|K_{n-r}\right\rangle & \hat{\mathcal{O}}^{r}\left|H_{\ell}\right\rangle=\omega^{-r \ell}\left|H_{\ell}\right\rangle .
\end{array}
$$

An irreducible unitary representation of the canonical Fourier-Kravchuk pairs $\left(\hat{\mathcal{F}}_{K}^{\alpha_{1}}, \hat{\mathcal{O}}^{k_{2}}\right)$ in the cyclic group $C_{D}$ can be obtained from (21) and from its Fourier transforms. The generators of this cyclic group are the $D \times D$ Weyl matrices [1]

$$
\begin{aligned}
\hat{\mathcal{F}}_{K}^{\alpha_{1}} \mapsto\left(\begin{array}{ccccc}
1 & 0 & 0 & \cdots & 0 \\
0 & \omega & 0 & \cdots & 0 \\
0 & 0 & \omega^{2} & \cdots & 0 \\
\vdots & \vdots & \vdots & \ddots & \vdots \\
0 & 0 & 0 & \cdots & \omega^{D-1}
\end{array}\right)=\sum_{n=0}^{D-1}\left|K_{n}\right\rangle \omega^{n}\left\langle K_{n}\right| \\
\hat{\mathcal{O}} \mapsto\left(\begin{array}{ccccc}
0 & 1 & 0 & \cdots & 0 \\
0 & 0 & 1 & \cdots & 0 \\
\vdots & \vdots & \vdots & \ddots & \vdots \\
0 & 0 & 0 & \cdots & 1 \\
1 & 0 & 0 & \cdots & 0
\end{array}\right)=\sum_{n=0}^{D-1}\left|K_{n}\right\rangle\left\langle K_{n+1}\right| .
\end{aligned}
$$

A convenient phase space representation of the Kravchuk oscillator can be constructed with the Schwinger operators [1]

$$
\hat{\mathcal{S}}_{\vec{k}}=\omega^{k_{1} k_{2} / 2} \hat{\mathcal{F}}_{K}^{\alpha_{k_{1}}} \hat{\mathcal{O}}^{k_{2}} \quad \vec{k}=\left(k_{1}, k_{2}\right) \in \mathbb{Z}_{D} \times \mathbb{Z}_{D} .
$$


The discrete-unitary-cyclic Schwinger operator basis (27) has the following properties which can be deduced from (21), (23) and (25),

$$
\begin{array}{ll}
\hat{S}_{\vec{k}} \hat{S}_{\vec{k}^{\prime}}=\omega^{\vec{k} \times \vec{k}^{\prime} / 2} \hat{S}_{\vec{k}+\vec{k}^{\prime}} & \text { (composition) } \\
\hat{S}_{\vec{k}} \hat{S}_{-\vec{k}}=\hat{S}_{\overrightarrow{0}}=I & \text { (unit and inverse) } \\
\left(\hat{S}_{\vec{k}} \hat{S}_{\vec{k}^{\prime}}\right) \hat{S}_{\vec{k}^{\prime \prime}}=\hat{S}_{\vec{k}}\left(\hat{S}_{\vec{k}^{\prime}} \hat{S}_{\vec{k}^{\prime \prime}}\right) & \text { (associativity) } \\
\hat{S}_{\vec{k}}^{\dagger}=\hat{S}_{-\vec{k}} & \text { (unitarity) } \\
\operatorname{Tr} \hat{S}_{\vec{k}}=D \delta_{\vec{k}, \overrightarrow{0}} & \text { (trace) }
\end{array}
$$

where $\vec{k} \times \vec{k}^{\prime} \equiv\left(k_{1} k_{2}^{\prime}-k_{2} k_{1}^{\prime}\right)$. The first property states that $\hat{\mathcal{S}}_{\vec{k}}$ is a projective representation of the Weyl-Heisenberg algebra generated by the operators $\hat{\mathcal{F}}_{K}^{\alpha_{1}}$ and $\hat{\mathcal{O}}$. When $D$ is prime, the representation is irreducible. The trace identity ensures the orthogonality of the basis.

\section{Action-angle Wigner function of the Kravchuk oscillator}

The finite $D$-dimensional Kravchuk oscillator Hamiltonian $\hat{\mathcal{H}}_{D}$ was connected with the fractional Fourier-Kravchuk operators $\hat{\mathcal{F}}_{K}^{\alpha_{k_{1}}}$ and $\mathcal{O}^{k_{2}}$ by (15) and (22). The Schwinger operators (27) are therefore a natural action-angle basis for the Kravchuk oscillator. We introduce now the discrete action-angle Wigner function [2,3] based on this discrete-unitary-cyclic Schwinger basis as the expectation value of the operator $[2,3]$

$$
\hat{\Delta}\left(n_{1}, n_{2}\right)=\frac{1}{D^{2}} \sum_{\vec{k} \in \mathbb{Z}_{D} \times \mathbb{Z}_{D}} \omega^{\vec{k} \times \vec{n}} \hat{\mathcal{S}}_{\vec{k}} \quad \vec{n}=\left(n_{1}, n_{2}\right) \in \mathbb{Z}_{D} \times \mathbb{Z}_{D}
$$

with $\omega=\exp (-2 \pi \mathrm{i} / D)$ as before. For a state $|\psi\rangle$, the Wigner function is

$$
\begin{aligned}
W\left(\psi \mid n_{1}, n_{2}\right) & =\left\langle\psi\left|\hat{\Delta}\left(n_{1}, n_{2}\right)\right| \psi\right\rangle \\
& \mapsto \frac{1}{D^{2}} \sum_{\vec{k} \in \mathbb{Z}_{D} \times \mathbb{Z}_{D}} \omega^{\vec{k} \times \vec{n}+k_{1} k_{2} / 2}\left\langle\psi\left|\hat{\mathcal{F}}_{K}^{\alpha_{k_{1}}} \hat{\mathcal{O}}^{k_{2}}\right| \psi\right\rangle .
\end{aligned}
$$

We choose the normalization such that $\sum_{n_{1}, n_{2}} W\left(\psi \mid n_{1}, n_{2}\right)=1$ for normalized states $\||\psi\rangle \|=$ 1. The Wigner function is periodic on the toroidal lattice $\mathbb{Z}_{D} \times \mathbb{Z}_{D}: W\left(\psi \mid n_{1}+D, n_{2}\right)=$ $W\left(\psi \mid n_{1}, n_{2}+D\right)=W\left(\psi \mid n_{1}, n_{2}\right)$. The properties of the kernel operator $\hat{\Delta}\left(n_{1}, n_{2}\right)$ in (30) have been studied in [2,3], where it was shown that it complies with all the fundamental properties of a phase space distribution function [9]. In the following we find the Wigner function of the action and angle eigenvector bases. We will be interested specifically in systems with one degree of freedom and choose $D$ to be prime.

When the state of the system is $K_{m}$, a Kravchuk eigenfunction of the Hamiltonian, from (27), (29) and (30), we find

$$
W\left(K_{m} \mid n_{1}, n_{2}\right)=\frac{1}{D} \delta_{n_{2}, m} .
$$

On the other hand, when the state is the Fourier transform over the energy values, $H_{\ell}$ in (19), the Wigner function is

$$
W\left(H_{\ell} \mid n_{1}, n_{2}\right)=\frac{1}{D} \delta_{n_{1}, \ell} .
$$

These result confirm $K_{m}$ in (32) as a finite-dimensional 'action-eigenstate' for which the angle distribution is uniform, and $H_{\ell}$ in (19) as a 'angle-eigenstate', independent of action. 
Another example of the discrete action-angle Wigner function is when $|\psi\rangle$ is a split Kravchuk state defined in analogy with the split photon states in quantum optics [10] (more commonly called 'Schrödinger cat' state) and given by

$$
\left|\psi_{m_{1}, m_{2}}^{\mathrm{SC}}\right\rangle=\frac{1}{\sqrt{2}}\left(\left|K_{m_{1}}\right\rangle \pm\left|K_{m_{2}}\right\rangle\right) \quad m_{1} \neq m_{2}
$$

The corresponding discrete Wigner function is the sum of the Wigner functions of the summands, plus an interference term (also called the 'smile of the cat' term):

$$
\begin{aligned}
W\left(\psi_{m_{1}, m_{2}}^{\mathrm{SC}} \mid n_{1}, n_{2}\right) & =\frac{1}{2 D}\left[\delta_{n_{2}, m_{1}}+\delta_{n_{2}, m_{2}}\right. \\
\pm \frac{2}{D} & \left.\sum_{r=-(D-1) / 2}^{(D-1) / 2} \omega^{r\left[n_{2}-\left(m_{1}+m_{2}\right) / 2\right]} \cos \left(\frac{2 \pi}{D} n_{1}\left(m_{1}-m_{2}\right)\right)\right]
\end{aligned}
$$

This Wigner function is of the same form as the well known continuous one; they exhibit two discrete- $\delta$ lines at $n_{2}=m_{1}$ and $n_{2}=m_{2}$, and a 'smile' function on the line in the middle of the two; the 'teeth' of the smile are the oscillations of the cosine function. The farther apart $m_{1}$ and $m_{2}$, the greater number of teeth in a length of the smile; in the discrete case (since $m_{1}-m_{2}$ is an integer) the smile is periodic and contains only a whole number of teeth.

The marginal distributions (or projections) of the split-state Wigner function (35) on the action and angle variables are respectively

$$
\begin{aligned}
& M_{2}\left(\psi_{m_{1}, m_{2}}^{\mathrm{SC}} \mid n_{2}\right)=\sum_{n_{1}=0}^{D-1} W\left(\psi_{m_{1}, m_{2}}^{\mathrm{SC}} \mid n_{1}, n_{2}\right)=\frac{1}{2}\left(\delta_{n_{2}, m_{1}}+\delta_{n_{2}, m_{2}}\right) \\
& M_{1}\left(\psi_{m_{1}, m_{2}}^{\mathrm{SC}} \mid n_{1}\right)=\sum_{n_{2}=0}^{D-1} W\left(\psi_{m_{1}, m_{2}}^{\mathrm{SC}} \mid n_{1}, n_{2}\right)=\frac{1}{D}\left[1 \pm \cos \left(\gamma_{0} n_{1}\left(m_{1}-m_{2}\right)\right)\right] .
\end{aligned}
$$

The marginal distribution of the action variable $n_{2}$ is consistent with $\left|\psi_{m_{1}, m_{2}}^{\mathrm{SC}}\right\rangle$ being a symmetric mixture of two pure action states. On the other hand, the marginal probability distribution in the angle (phase) variable $n_{1}$ contains the interference between the pure action states. It is clear that the Wigner function and the marginal probabilities in (35)-(37) are properly normalized.

\section{The quantum phase operator for the Kravchuk oscillator and its time evolution}

Let us recapitulate the reasons for the construction of well defined action (Hamiltonian) and angle operators, since it is the latter which is not satisfactorily defined in the continuum case. In $[2,3]$ it was shown that a quantum action-angle formalism is equivalent to the formulation of the quantum phase problem in phase space. The search for the quantum phase of the standard harmonic oscillator is obstructed by the fact that the standard oscillator Hamiltonian (which we symbolically denote by $\hat{\mathcal{H}}_{\infty}$ ) is invariant under the Fourier transformation.

The solution to the phase operator problem for the quantum harmonic oscillator is made possible by working in finite-dimensional spaces, for which the canonical algebraic formulation provides the unitary canonical pair. Of particular interest to this approach are the Hamiltonians for which a unitary canonical partner exists for all dimension $D$. The Kravchuk oscillator Hamiltonian $\hat{\mathcal{H}}_{D}$ in (13) is one example in the set of such Hamiltonians, whose discrete spectrum is naturally equidistant. We also note here that the Kravchuk oscillator eigenstates are the natural basis for the finite-dimensional 'truncated' Hilbert space formalism introduced by Pegg and Barnett [11]. 
We now examine the time evolution of the quantum phase operator $\mathcal{O}^{k}$ in (22) generated by the Kravchuk oscillator $\hat{\mathcal{H}}_{D}$, identified via [3] with its unitary canonical partner. From (15) we find its time evolution to be

$$
\hat{\mathcal{O}}^{k}(t)=\mathrm{e}^{\mathrm{i} \hat{\mathcal{H}}_{D} t} \hat{\mathcal{O}}^{k} \mathrm{e}^{-\mathrm{i} \hat{\mathcal{H}}_{D} t}
$$

Its matrix elements in the Kravchuk basis can be found using (25); they are

$$
\begin{aligned}
\left\langle K_{n}\left|\hat{\mathcal{O}}^{k}(t)\right| K_{m}\right\rangle & =\mathrm{e}^{\mathrm{i}\left(\mu_{m}-\mu_{n}\right) t}\left\langle K_{n}\left|\hat{\mathcal{O}}^{k}\right| K_{m}\right\rangle \\
& =\mathrm{e}^{\mathrm{i} k t} \delta_{m, n+k} \quad n, m, k \in \mathbb{Z}_{D} .
\end{aligned}
$$

Therefore, the time evolution of the exponential phase (angle) operator is given by the classical result.

We now examine the time dependence of the action-angle Wigner function for the Kravchuk oscillator. The time dependence of the Wigner function is calculated by

$$
W\left(\psi \mid n_{1}, n_{2} ; t\right)=\left\langle\psi\left|\mathrm{e}^{\mathrm{i} \hat{\mathcal{H}}_{D} t} \Delta(\vec{n}) \mathrm{e}^{-\mathrm{i} \hat{\mathcal{H}}_{D} t}\right| \psi\right\rangle .
$$

It can be directly checked that the Wigner function in pure action state has no time depedence. This result can be obtained very easily once the state $K_{m}$ is inserted into (40) instead of (30). Since the time evolution of the pure states does not affect the Wigner function, we will instead show the time dependent action-angle Wigner function initially prepared in a split (Schrödinger cat) state $\left|\psi_{m_{1}, m_{2}}^{\mathrm{SC}}\right\rangle$ in (34). A simple calculation yields

$$
\begin{aligned}
W\left(\psi_{m_{1}, m_{2}}^{\mathrm{SC}} \mid n_{1}, n_{2} ; t\right) & =\frac{1}{2 D}\left[\delta_{n_{2}, m_{1}}+\delta_{n_{2}, m_{2}}\right. \\
\pm \frac{2}{D} & \left.\sum_{r=-(D-1) / 2}^{(D-1) / 2} \omega^{r\left[n_{2}-\left(m_{1}+m_{2}\right) / 2\right]} \cos \left(\left(\gamma_{0} n_{1}-t\right)\left(m_{1}-m_{2}\right)\right)\right]
\end{aligned}
$$

for which we obtain the marginal distributions

$$
\begin{aligned}
& M_{2}\left(\psi_{m_{1}, m_{2}}^{\mathrm{SC}} \mid n_{2} ; t\right)=\frac{1}{2}\left(\delta_{n_{2}, m_{1}}+\delta_{n_{2}, m_{2}}\right) \\
& M_{1}\left(\psi_{m_{1}, m_{2}}^{\mathrm{SC}} \mid n_{1} ; t\right)=\frac{1}{D}\left[1 \pm \cos \left(\left(\gamma_{0} n_{1}-t\right)\left(m_{1}-m_{2}\right)\right)\right]
\end{aligned}
$$

where both distributions are appropriately normalized. We observe that the action distribution (42) is time independent, whereas the angular distribution rotates (43) with unit frequency (as assumed in (13)) as

$$
\theta_{n_{1}}(t)=\theta_{n_{1}}(0)-t \quad \text { where } \quad \theta_{n_{1}}(0)=\gamma_{0} n_{1} .
$$

\section{The continuum limit}

As shown in (14), the Kravchuk functions have a well defined limit, when $D \rightarrow \infty$ [4-6], to the continuous Hermite functions of the standard quantum oscillator. Hence, in this limit the matrix elements of the unitary operator $\hat{\mathcal{O}}$ in (39) are also well defined. However, the Hamiltonian $\hat{\mathcal{H}}_{D}$ has a singular behaviour in this limit, at which it becomes a fixed point in the Fourier automorphism generated by $\hat{\mathcal{F}}$.

There are several ways to obtain continuous limits for the discrete Wigner function in (29), (30). One way is to replace in the operator (29) the discrete index $n_{1}$ by the continuous one $\theta=\lim _{D \rightarrow \infty} 2 \pi\left[n_{1}-(D-1) / 2\right] / D$ and keep the other one discrete as $J=n_{2}$, so that $-\pi \leqslant \theta \leqslant \pi$ becomes an angle. Let us also similarly use a symmetric range for $J$ as $-(D-1) / 2 \leqslant J \leqslant(D-1) / 2$. We write the operator

$$
\hat{\Delta}_{D}(\theta, J)=\frac{1}{2 \pi D} \sum_{\vec{k}} \mathrm{e}^{\mathrm{i}\left(2 \pi k_{1} J / D-k_{2} \theta\right)} \hat{\mathcal{S}}_{\vec{k}}
$$


to find the limit of the Wigner function

$$
W(\psi \mid \theta, J)=\lim _{D \rightarrow \infty}\left\langle\psi\left|\hat{\Delta}_{D}(\theta, J)\right| \psi\right\rangle .
$$

We have changed the normalization of (45) in compliance with the continuum Wigner function normalization [9], given by $\int_{-\pi}^{\pi} \mathrm{d} \theta \sum_{J=-\infty}^{\infty} W(\psi \mid \theta, J)=1$. Another adaptation is that the sum over $\vec{k}$ in (45) will also be performed over the symmetric range $-\frac{1}{2}(D-1) \leqslant k_{1}, k_{2} \leqslant$ $\frac{1}{2}(D-1)$. Since we confine our attention to systems with $D$ prime, the limit is understood to occur over the range of primes.

In the action (Kravchuk) basis, where $\psi=K_{m}$, we can use (25) and (29) to perform the following straightforward calculation:

$$
\begin{aligned}
W\left(K_{m} \mid \theta, J\right) & =\lim _{D \rightarrow \infty} \frac{1}{2 \pi} \sum_{k=-\frac{1}{2}(D-1)}^{\frac{1}{2}(D-1)} \mathrm{e}^{-\mathrm{i} k \theta}\left\langle K_{m}\left|\hat{\mathcal{O}}^{-k / 2}\right| K_{J}\right\rangle\left\langle K_{J}\left|\hat{\mathcal{O}}^{-k / 2}\right| K_{m}\right\rangle \\
& =\lim _{D \rightarrow \infty} \frac{1}{2 \pi} \sum_{k=-\frac{1}{2}(D-1)}^{\frac{1}{2}(D-1)} \mathrm{e}^{-\mathrm{i} k \theta}\left\langle K_{m} \mid K_{J-k / 2}\right\rangle\left\langle K_{J+k / 2} \mid K_{m}\right\rangle \\
& =\lim _{D \rightarrow \infty} \frac{1}{2 \pi D} \sum_{k=-\frac{1}{2}(D-1)}^{\frac{1}{2}(D-1)} \mathrm{e}^{2 \pi \mathrm{i} k(J-m) / D}=\frac{1}{2 \pi} \delta_{J, m} .
\end{aligned}
$$

In the above derivation, in (47) it must be noted that the states $K_{J-k / 2}$ and $K_{J+k / 2}$ are fractionally shifted Kravchuk states; for a general discussion on the finite-dimensional fractionally shifted orthonormal sets, see [2]. Equation (48) leads to the expected result that the phase is uniformly distributed over the $2 \pi$-range of $\theta$ and the action variable $J$ has a distribution given by the discrete delta function.

For split Kravchuk states (34), where now $m_{1}, m_{2} \in \mathbb{Z}, m_{1} \neq m_{2}$, we calculate the time dependence of the continuous Wigner function. Using (34) we find [3]

$$
\begin{aligned}
W\left(\psi_{m_{1}, m_{2}}^{\mathrm{SC}} \mid \theta, J ; t\right) & =\lim _{D \rightarrow \infty} \frac{1}{2 \pi} \sum_{k=-\frac{1}{2}(D-1)}^{\frac{1}{2}(D-1)} \mathrm{e}^{-\mathrm{i} k \theta}\left\langle\psi \mid K_{J-k / 2}\right\rangle\left\langle K_{J+k / 2} \mid \psi\right\rangle \\
& =\frac{1}{4 \pi}\left\{\delta_{J, m_{1}}+\delta_{J, m_{2}} \pm 2 \int_{-\pi}^{\pi} \frac{\mathrm{d} \gamma}{2 \pi} \mathrm{e}^{\mathrm{i} \gamma\left[J-\left(m_{1}+m_{2}\right) / 2\right]} \cos \left[(\theta-t)\left(m_{1}-m_{2}\right)\right]\right\} .
\end{aligned}
$$

Finally, the marginal distributions in the action and phase variables are

$$
\begin{aligned}
& M_{2}\left(\psi_{m_{1}, m_{2}}^{\mathrm{SC}} \mid J\right)=\int_{-\pi}^{\pi} \mathrm{d} \theta W\left(\psi_{m_{1}, m_{2}}^{\mathrm{SC}} \mid \theta, J\right)=\frac{1}{2}\left[\delta_{J, m_{1}}+\delta_{J, m_{2}}\right] \\
& M_{1}\left(\psi_{m_{1}, m_{2}}^{\mathrm{SC}} \mid \theta\right)=\sum_{J=-\infty}^{\infty} W\left(\psi_{m_{1}, m_{2}}^{\mathrm{SC}} \mid \theta, J\right)=\frac{1}{2 \pi}\left[1 \pm \cos \left((\theta-t)\left(m_{1}-m_{2}\right)\right)\right]
\end{aligned}
$$

which are the correct distributions for a split state [10]. The action distribution is time independent, whereas the angular distribution advances as $\theta(t)=\theta-t$. This implies that the action-angle Wigner function traces in time a circle in phase space of radius $J$ and phase $\theta(t)$ rotating uniformly with unit frequency.

\section{Conclusions}

We have examined the action-angle quantum phase space formulation of the discrete, finite harmonic oscillator in the Kravchuk basis. It was shown that the Kravchuk oscillator 
Hamiltonian has a unitary canonical partner in the standard Fourier sense for all finite dimensions. The infinite-dimensional limit yields another canonical algebraic approach to the quantum phase problem of the harmonic oscillator [2,3]. We suggest [12] that the absence of the standard quantum oscillator phase operator is connected with the fact that its Hamiltonian is invariant under the Fourier transformation, which manifests itself as a singularity in the $D \rightarrow \infty$ limit.

The direct discretization method has much to offer in the understanding of discrete quantum mechanics. In particular, a canonical algebraic extension of the Kravchuk formalism to other cases has not been studied yet. We expect that this extension, which was the basic motivation of this work, should bring additional insight to the discretization process.

\section{Acknowledgments}

The authors are grateful to the Centro Internacional de Ciencias for hospitality in Cuernavaca, and project DGAPA-UNAM IN104198 'Optica Matemática' for the support of TH. KBW thanks Bilkent University for kind attention and expenses. Both authors are thankful to N M Atakishiyev for useful discussions. The figures were prepared by Luis Edgar Vicent, at CCF-UNAM.

\section{References}

[1] Weyl H 1931 The Theory of Groups in Quantum Mechanics (New York, Dover) Schwinger J 1960 Proc. Natl Acad. Sci. 46883

Schwinger J 1960 Proc. Natl Acad. Sci. 461461

[2] Hakioğlu T 1998 J. Phys. A: Math. Gen. 316975

[3] Hakioğlu T 1999 J. Phys. A: Math. Gen. 324111

[4] Nikiforov A F and Uvarov V B 1988 Special Functions of Mathematical Physics (Basle: Birkhäuser)

[5] Atakishiyev N M and Suslov S K 1991 Theor. Math. Phys. 851055

[6] Atakishiyev N M and Wolf K B 1997 J. Opt. Soc. Am. A 141467

[7] Atakishiyev N M, Vicent L E and Wolf K B 1999 J. Comput. Appl. Math. 10773

[8] Erdélyi A, Magnus W, Oberhettinger F and Tricomi F G 1953 Higher Transcendental Functions (Bateman Manuscript Project vol 2) (New York: McGraw-Hill)

Koekoek R and Swarttouw R 1994 The Askey-scheme of hypergeometric orthogonal polynomials and its qanalogue Delft University of Technology, Faculty of Technical Mathematics and Informatics Report No 94-05

[9] Hillery M, O'Connell R F, Scully M O and Wigner E P 1984 Phys. Rep. 106121

[10] Noh J W, Fougères A and Mandel L 1992 Phys. Rev. A 45424 Hakioğlu T 1999 Phys. Rev. A 591586

[11] Pegg D T and Barnett S M 1988 Europhys. Lett. 6483 Pegg D T and Barnett S M 1989 Phys. Rev. A 391665

[12] Hakioğlu T and Tepedelenlioğlu E 2000 J. Phys. A: Math. Gen. in press 